PROCEEDINGS OF THE

AMERICAN MATHEMATICAL SOCIETY

Volume 89. Number 3, November 1983

\title{
THE ASYMPTOTIC NORMING PROPERTY AND MARTINGALE CONVERGENCE
}

D. VAN DULST

\begin{abstract}
A martingale proof is given of the result of R. G. James and A. Ho in [3] that the asymptotic norming property implies the Radon-Nikodym property.
\end{abstract}

In [3] R. C. James and A. Ho introduced and studied the asymptotic norming property (ANP). Their main result is that a Banach space with the ANP has the RNP (= Radon-Nikodym property). This is of considerable interest because there exist separable spaces having the ANP (and therefore the RNP) which cannot be isomorphically embedded in separable duals $[3,4]$. The proof of the theorem, however, is rather complicated. By using martingale theory we give a much shorter proof that directly extends Chatterji's methods [1].

Various formally different definitions of the ANP are given in [3]. There is no need here to state them and to show their equivalence. In fact our proof works for the formally weakest one, which we recall below. Let $X$ be a Banach space. We denote the unit ball $\{x:\|x\| \leqslant 1\}$ by $B(X)$ and the unit sphere $\{x:\|x\|=1\}$ by $S(X)$. Our other terminology is standard. For notions unexplained we refer to [2]. A norming set for $X$ is a subset $\Phi$ of $B\left(X^{*}\right)$ such that $\|x\|=\sup \left\{\left\langle x, x^{*}\right\rangle: x^{*} \in \Phi\right\}$ for every $x \in X$. A sequence $\left(x_{n}\right) \subset S(X)$ is said to be asymptotically normed by $\Phi$ if for each $\varepsilon>0$ there exist $x^{*} \in \Phi$ and $N \in \mathbf{N}$ such that $\left\langle x_{n}, x^{*}\right\rangle>1-\varepsilon$ whenever $n \geqslant N$.

DEFINITION. $X$ has the asymptotic norming property (ANP) if after some equivalent renorming there exists a subset $\Phi \subset B\left(X^{*}\right)$ with the following properties:

(i) $\Phi$ is a norming set for $X$;

(ii) for every sequence $\left(x_{n}\right) \subset S(X)$ which is asymptotically normed by $\Phi$ the set $\cap_{n=1}^{\infty} K_{n}$ is nonempty, where $K_{n}=\overline{\operatorname{co}}\left\{x_{i}: i \geqslant n\right\}(n=1,2, \ldots)$.

We now recall a basic result of Chatterji [1]. A Banach space $X$ has the RNP iff every $X$-valued uniformly bounded martingale defined on $[0,1]$ with Lebesgue measure $\lambda$ converges almost surely (a.s.) in norm. The following lemma formulates a criterion for a.s. convergence of uniformly bounded martingales in dual spaces and will be useful later. We sketch a proof for completeness, although essentially this lemma is contained in [1].

LEMMA 1. Let $X$ be a Banach space and let $\left(f_{n}, \Sigma_{n}, n \in \mathbf{N}\right)$ be a uniformly bounded $X^{*}$-valued martingale on $[0,1]$. Then the following holds.

Received by the editors November 23, 1982.

1980 Mathematics Subject Classification. Primary 46B22. 
(i) There exists a uniformly bounded $w^{*}$-measurable function $f:[0,1] \rightarrow X^{*}$ with the property that for every $x \in X$ there is a $\lambda$-null set $N_{x}$ such that $\lim _{n \rightarrow \infty}\left\langle x, f_{n}(\omega)\right\rangle=$ $\langle x, f(\omega)\rangle$ whenever $\omega \notin N_{x}$.

(ii) $\left(f_{n}\right)$ converges a.s. in norm iff there exists a $\lambda$-essentially separably valued $f$ satisfying (i). In this case $f$ is the a.s. limit of $\left(f_{n}\right)$.

Proof. For every $\omega \in[0,1]$ let $f(\omega)$ be a $w^{*}$-limit point of $\left(f_{n}(\omega)\right)$. Since for each $x \in X$ the scalar-valued martingale $\left(\left\langle x, f_{n}\right\rangle, \Sigma_{n}, n \in \mathbf{N}\right)$ converges a.s., and since, obviously, $\langle x, f\rangle$ is the only possible limit, (i) follows. For the proof of (ii) suppose there exists a $\lambda$-essentially separably valued $f$ as in (i). Let $W$ denote a separable subspace of $X^{*}$ that $\lambda$-essentially contains the ranges of $f$ and those of all $f_{n}$, and let $\left(x_{n}\right) \subset B(X)$ be a norming sequence for $W$. Since the functions $\left\langle x_{n}, f\right\rangle$ are all measurable it follows by a standard argument (cf. [2, Corollary II, 1.4]) that $f$ is measurable and therefore Bochner integrable. To show that $\left(f_{n}\right)$ converges a.s. it suffices to prove that $f_{n}=\mathcal{E}\left(f \mid \Sigma_{n}\right)(n=1,2, \ldots)$ [2, Corollary V, 2.2 and Theorem $\mathrm{V}, 2.8$ ]. Let $n \in \mathbf{N}, A \in \Sigma_{n}$ and $x \in X$ be arbitrary. Then we have

$$
\begin{aligned}
\left\langle x, \int_{A} f_{n} d \lambda\right\rangle & =\int_{A}\left\langle x, f_{n}\right\rangle d \lambda=\lim _{m \rightarrow \infty} \int_{A}\left\langle x, f_{m}\right\rangle d \lambda \\
& =\int_{A}\langle x, f\rangle d \lambda=\left\langle x, \int_{A} f d \lambda\right\rangle .
\end{aligned}
$$

It follows that $\int_{A} f_{n} d \lambda=\int_{A} f d \lambda$, which means $\delta\left(f \mid \Sigma_{n}\right)=f_{n}$. Clearly $f$ is the a.s. limit of $\left(f_{n}\right)$. The "only if" part of (ii) is obvious.

REMARK. The well-known fact that separable duals and reflexive spaces have the RNP is an immediate consequence of Lemma 1 and Chatterji's result.

The second tool we need is a lemma of Neveu [5, Lemma V, 2.9].

LeMma 2. For each $m \in \mathbf{N}$ let $\left(f_{n}^{(m)}, \Sigma_{n}, n \in \mathbf{N}\right)$ be a real-valued submartingale on some probability space and assume the sequence $\left(\sup _{m \in \mathbf{N}} f_{n}^{(m)+}\right)_{n=1}^{\infty}$ is $L^{1}$-bounded. Then

(i) each of the submartingales $\left(f_{n}^{(m)}\right)_{n=1}^{\infty}$ converges a.s. to an integrable limit $f^{(m)}$ $(m=1,2, \ldots)$, and

(ii) $\sup _{m \in \mathbf{N}} f_{n}^{(m)} \rightarrow \sup _{m \in \mathbf{N}} f^{(m)}$ a.s. as $n \rightarrow \infty$.

We are now ready for the main result [3, Theorem 1.8].

THEOREM. A Banach space $X$ with the ANP has the RNP.

Proof. Since the RNP is separably determined and invariant for isomorphisms and since the ANP is clearly inherited by subspaces, we may assume that $X$ is separable and that the given norm on $X$ admits a set $\Phi \subset B\left(X^{*}\right)$ as in the Definition. We may further assume $\Phi$ is countable. ( $\Phi$ contains a countable norming subset $\Phi^{\prime}$ since $X$ is separable and every sequence in $S(X)$ asymptotically normed by $\Phi^{\prime}$ is also asymptotically normed by $\Phi$.) As in [3] we now introduce on the linear subspace spanned by $\Phi$ a new norm, namely the gauge of $\operatorname{co}\{\Phi \cup-\Phi\}$. If $Y$ denotes the completion of $\operatorname{sp} \Phi$ for this new norm, then it is easy to see that $X$ is isometric to a subspace of $Y^{*}$ and $X$, with its given norm, satisfies (i) and (ii) in the 
Definition with $\Phi$ equal to $B(Y)$. (Observe that a sequence in $S(X)$ asymptotically normed by $B(Y)$ has a subsequence asymptotically normed either by $\Phi$ or by $-\Phi$.) Notice also that $Y$ is separable.

Now let $\left(f_{n}, \Sigma_{n}, n \in \mathbf{N}\right)$ be a uniformly bounded $X$-valued martingale defined on $[0,1]$. What must be shown is that $\left(f_{n}\right)$ converges a.s. in norm. We regard $\left(f_{n}\right)$ as a $Y^{*}$-valued martingale and apply both lemmas. Let $f$ be as in Lemma 1(i), and let $\left(y_{m}\right)$ be a dense sequence in $B(Y)$. Lemma 2 applied with $f_{n}^{(m)}=\left\langle y_{m}, f_{n}\right\rangle(n, m \in \mathbf{N})$ yields that

$$
\sup _{m \in \mathbf{N}}\left\langle y_{m}, f_{n}\right\rangle=\left\|f_{n}\right\| \rightarrow \sup \left\langle y_{m}, f\right\rangle=\|f\| \text { a.s. }
$$

Let $N$ be a $\lambda$-null set such that

$$
\left\langle y, f_{n}(\omega)\right\rangle \rightarrow\langle y, f(\omega)\rangle .
$$

and

$$
\left\|f_{n}(\omega)\right\| \rightarrow\|f(\omega)\|
$$

hold for all $\omega \notin N$ and all $y \in\left\{y_{1}, y_{2}, \ldots\right\}$. Now fix $\omega \notin N$ and assume $\|f(\omega)\|=1$ (the case $f(\omega)=0$ is trivial). For any $\varepsilon>0$ choose $y_{m}$ so that $\left\langle y_{m}, f(\omega)\right\rangle>1-\varepsilon$. Then also $\left\langle y_{m}, f_{n}(\omega)\right\rangle>1-\varepsilon$ for sufficiently large $n \in \mathbf{N}$. It follows now that the sequence $\left(f_{n}(\omega) /\left\|f_{n}(\omega)\right\|\right) \subset S(X)$ is asymptotically normed by $B(Y)$. Therefore

$$
K:=\bigcap_{n=1}^{\infty} K_{n} \neq \varnothing, \text { where } K_{n}=\overline{\operatorname{co}}\left\{\frac{f_{i}(\omega)}{\left\|f_{i}(\omega)\right\|}: i \geqslant n\right\} \text {. }
$$

It is now clear from (1) and (2) that $K=\{f(\omega)\}$. Since $K \subset X$ and $X$ is separable, we have now shown that $f$ is $\lambda$-essentially separably valued, and this concludes the proof by Lemma 1(ii).

\section{REFERENCES}

1. S. D. Chatterji, Martingale convergence and the Radon-Nikodym theorem in Banach spaces, Math. Scand. 22 (1968), 21-4l.

2. J. Diestel and J. J. Uhl, Jr., Vector measures, Math. Surveys, no. 15, Amer. Math. Soc., Providence, R. I., 1977.

3. R. C. James and Aggie Ho, The asymptotic-norming and Radon-Nikodym properties for Banach spaces, Ark. Mat. 19 (1981), 53-70.

4. P. McCartney and R. O'Brien, A separable Banach space with the Radon-Nikodym property which is not isomorphic to a subspace of a separable dual, Proc. Amer. Math. Soc. 78 (1980), 40-42.

5. J. Neveu, Discrete-parameter martingales, North-Holland, Amsterdam, 1975.

Department of Mathematics, University of AmSTERdam, 1018 WB Amsterdam, The NetherLANDS 